\title{
INNOVATION IN THE CLASSROOM: \\ A TRANSPORTATION GEOTECHNICS APPLICATION OF DESKTOP LEARNING MODULES TO PROMOTE INDUCTIVE LEARNING
}

Final Report

By

\author{
David S. Hurwitz \\ H. Benjamin Mason \\ Rachel Adams \\ Kamilah Buker \\ Richard Slocum \\ Oregon State University
}

\author{
For \\ Kiewit Center for Infrastructure and Transportation Research \\ Oregon State University
}




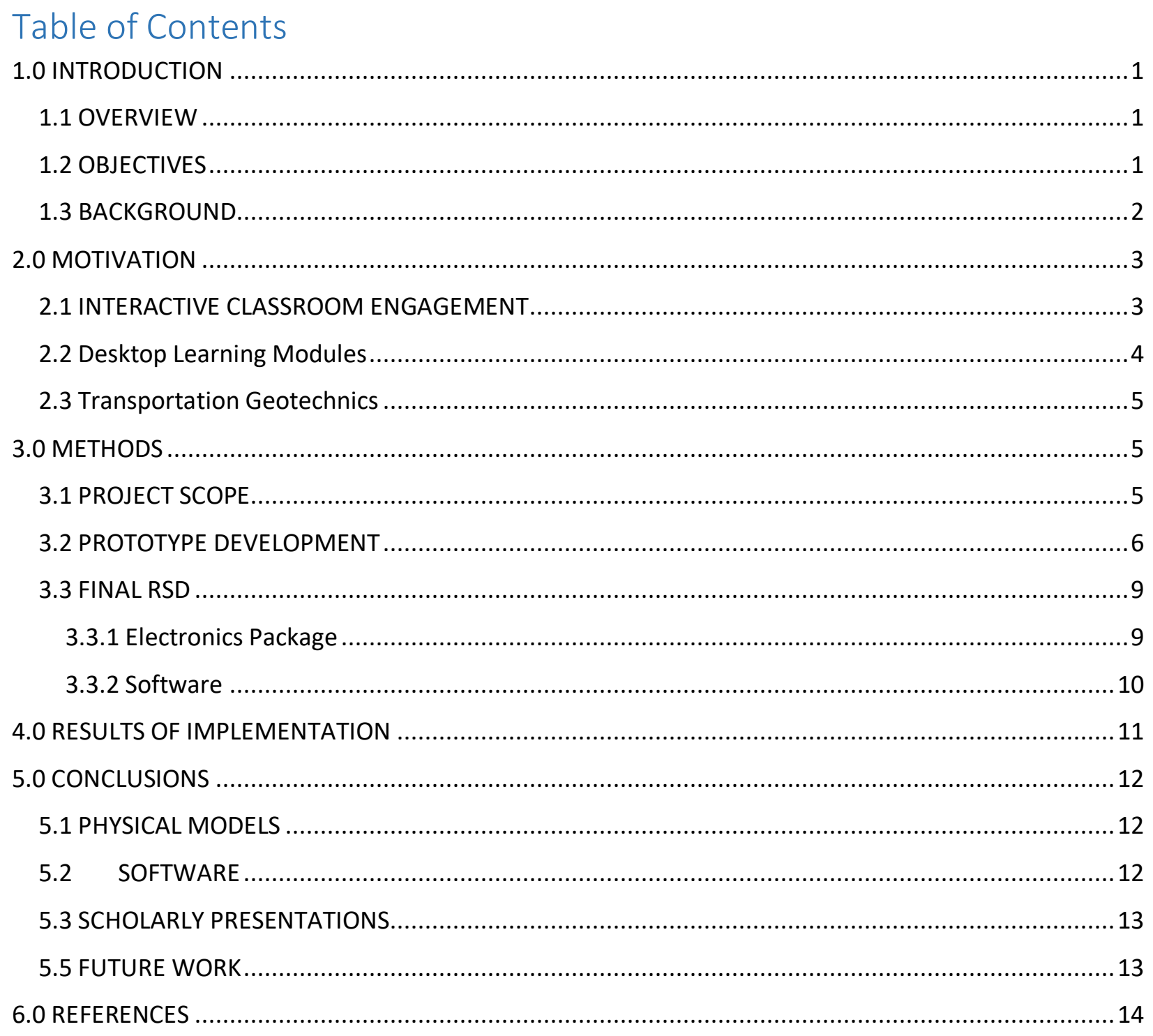




\section{List of Tables}

Table 1. Specifications of RSD components. The noted size and mass for each component is estimated from a sample set of RSD supplies. Each finalized model will have slightly different specifications due to construction processes.

Table 2. The components for the electronics package were selected to minimize the cost of the overall system.

\section{List of Figures}

Figure 1. Examples of transportation infrastructure systems subjected to extreme loading: (a) Road damage caused by soil liquefaction during the 2007 Niigata-Chuetsu Oki, Japan Earthquake (USGS 2007), (b) Entire road networks washed out during the 2012 Hurricane Sandy disaster (GEER 2014), (c) Kesen Bridge damaged during the 2011 Great East Japan Earthquake and Tsunami (Unjoh 2012), (d) Road washout from the 2015 South Carolina floods (State 2015).

Figure 2. The inductive approach for teaching response spectra: (a) a DLM for simulating response spectra, (b) physical measurements from the DLM, (c) the theory behind the DLM and measurements; i.e., equation of motion and vibrational properties, (d) the numerical method used to solve the equation of motion, and (e) the displacement response spectrum.

Figure 3. Development of finalized RSDs. Spherical weights were painted (left) and later applied to springs that had been welded to plates and painted (right)

Figure 4. Various spring and weight arrangements investigated to determine the best configuration for the finalized RSDs.

Figure 5. Finalized RSDs without instrumentation. The model on the left demonstrates the effect of varying structure weights and on the right the effect of varying stiffness of the structure.

Figure 6. The preliminary instrumented RSD (left) is shown with a sample of 3-axis accelerations exerted on each of the spring masses plotted with time (right). Accelerometers fixed to the model weights record and plot the response when the device is subjected to motion.

Figure 7. The protoboard design for each electronics package is used to interface the microcontrollers with the accelerometer connectors.

Figure 8. Finalized DLMs with instrumentation (left), and the Python GUI for data acquisition visualizes the accelerometer data in real time (right). 


\subsection{INTRODUCTION}

\subsection{OVERVIEW}

In many undergraduate engineering programs, students do not synthesize content learned from multiple courses until a capstone senior design course. As an example, in undergraduate civil engineering programs, transportation engineering concepts (e.g., geometric alignment, asphalt design procedures) and geotechnical engineering concepts (e.g., shear strength of soils, soil compaction) are not often synthesized until senior design, if then. As a result, concepts learned in multiple upper-division engineering courses, as well as other required courses, often seem disparate to students. In reality, engineers are required to synthesize concepts learned in a broad number of their courses to develop creative solutions to unique problems. This will particularly be true for the engineer of the future, who will need to develop unique solutions to problems caused by climate change and increasing global population, among others.

\subsection{OBJECTIVES}

We believe that a synthesis of concepts sourced from seemingly disparate courses needs to occur earlier in the engineering curriculum. We hypothesize that the curriculum change will have multiple benefits; namely, students will learn how to become more creative problem solvers, and will be more motivated by their coursework. We propose using Desktop Learning Modules (DLMs), combined with inductive learning and interactive learning techniques, to test our hypothesis.

Following the foregoing hypothesis statement, there is a research need to understand how DLMs, coupled with inductive and interactive learning techniques, will help students synthesize seemingly disparate course concepts. A related educational need is developing a community of educators who adopt evidence-based instructional practices. Accordingly, the objective of this proposed work is to address the identified research and educational needs by developing a novel transportation geotechnics DLM.

Although the interdisciplinary field of transportation geotechnics was the focus of this project, the results are transferable to other engineering domains. A particular focus was the response of transportation infrastructure to extreme events (e.g., hurricanes, earthquakes, tsunamis, floods), because engineers of the future, throughout the United States, need to learn how to protect existing infrastructure and design new infrastructure to withstand extreme loading. Within a transportation geotechnics DLM, we can replicate different engineering scenarios and help students answer important questions; for instance: What is the response of a coastal bridge during a large earthquake? How does the response of the bridge change during an ensuing tsunami? How can we change the bridge design to improve its performance during natural hazards? Figure 1 shows damage to transportation infrastructure caused by various natural disasters.

The foregoing questions exemplify how a DLM could promote an inductive learning style, whereby students first ask important questions, then physically observe a phenomenon, and finally develop the supporting theory. In addition, DLMs naturally promote interactive classrooms, where the students develop important questions to be answered, and then determine the steps required to answer them with group discussion. Engineering education specialists have suggested that inductive learning is an optimal style for engineering education and that the interactive classroom promotes retention of critical course concepts (Felder 2002). 


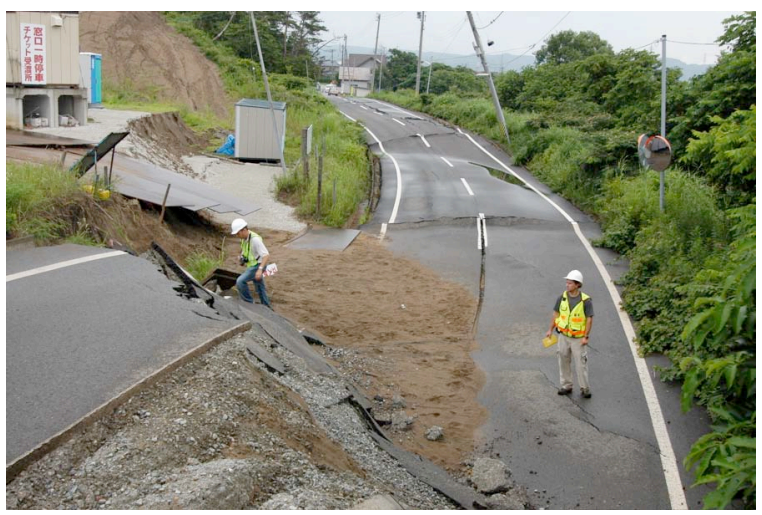

(a)

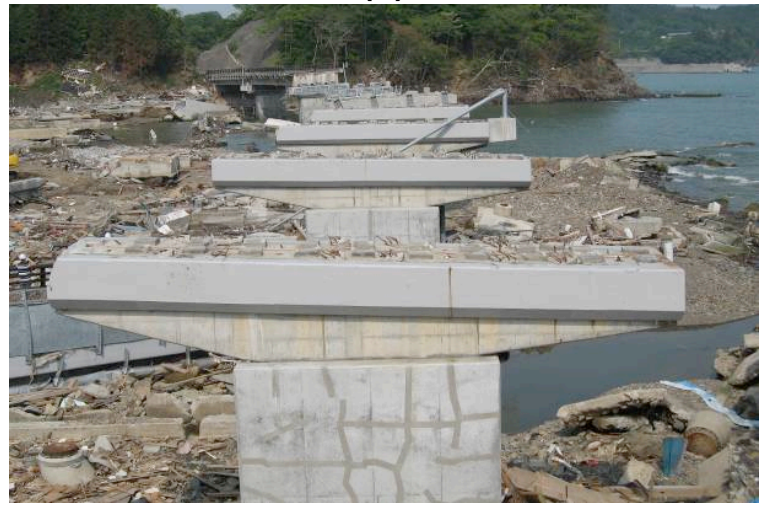

(c)

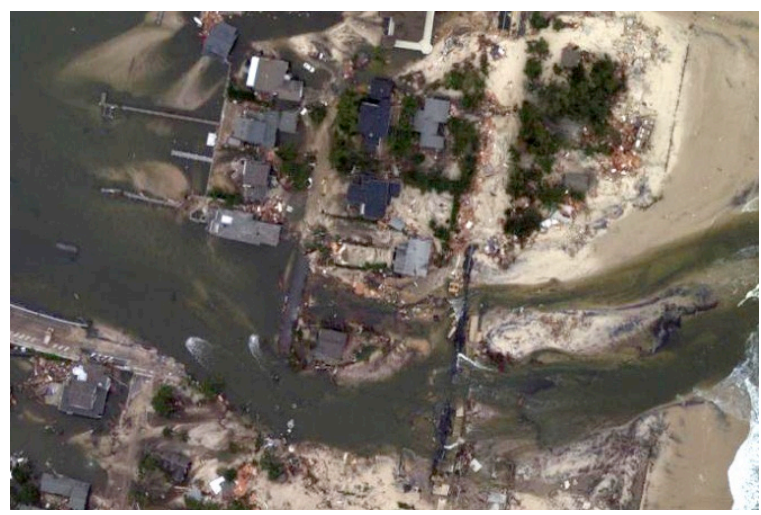

(b)

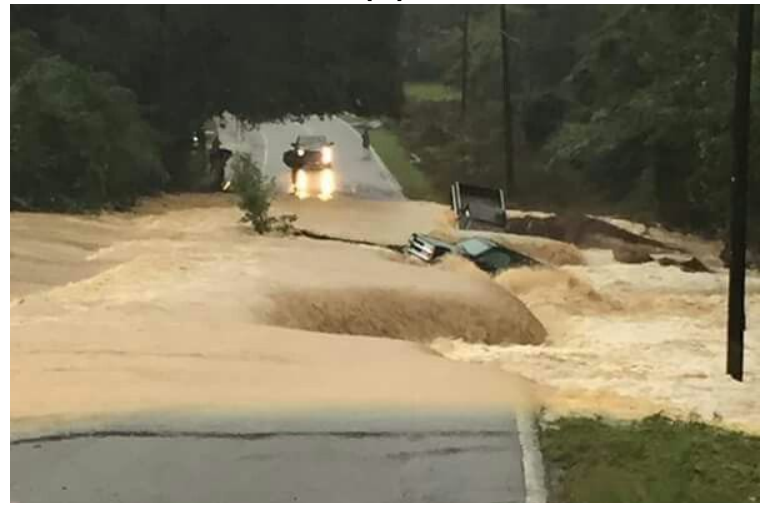

(d)

Figure 1. Examples of transportation infrastructure systems subjected to extreme loading: (a) Road damage caused by soil liquefaction during the 2007 Niigata-Chuetsu Oki, Japan Earthquake (USGS 2007), (b) Entire road networks washed out during the 2012 Hurricane Sandy disaster (GEER 2014), (c) Kesen Bridge damaged during the 2011 Great East Japan Earthquake and Tsunami (Unjoh 2012), (d) Road washout from the 2015 South Carolina floods (State 2015).

The transportation geotechnics DLM represents a first step towards the meaningful incorporation of inductive and interactive learning approach in courses that include transportation geotechnics content.

\subsection{BACKGROUND}

In an engineering classroom, inductive learning begins by engaging students with specific examples or activities and providing students with the opportunity to observe and interpret the resulting patterns; thereby, students can construct generalizable understanding (e.g., Felder and Silverman 1988). At the heart of inductive learning theory is the philosophy of constructivism, which posits that there is no objective reality (e.g., Biggs 1996); accordingly, a person must define her own reality to understand her experiences. A contrasting theory to constructivism is positivism, which states that there is an objective reality independent of human perception (e.g., Ayer 1966). The theory of positivism lends itself to deductive learning. In an engineering classroom, deductive learning requires that students learn general theory first, and then apply the theory to, for example, engineering design scenarios. Engineering courses have been traditionally taught using a deductive learning approach (Prince and Felder 2006; Felder 2012).

A literature synthesis on the effectiveness of inductive learning by Prince and Felder (2006) showed that inductive learning approaches are at least as effective, if not more effective than deductive learning approaches, for improving student learning outcomes. To further understand the effectiveness of the inductive learning approach, Prince and Felder (2006) listed six common instruction techniques to 
promote inductive learning in engineering classrooms: (1) guided inquiry, (2) problem-based, (3) projectbased, (4) case-based, (5) discovery, and (6) just-in-time teaching.

In the guided inquiry technique, the instructor poses questions and problems or provides observations, and then the students are guided to the answers or explanations by working in groups, or by classroom discussions (Lee 2012). The problem-based, project-based, and case-based techniques are similar. In the problem-based approach, students are given a complex and often ill-defined problem, and they are required to develop solutions by themselves, in groups, and in class discussion (Barrows and Tamblyn 1980; Yadav et al. 2011). The guided inquiry technique and the problem-based technique are similar; however, the amount of instructor guidance is usually much greater for the guided inquiry technique, and especially at first, when students are becoming acquainted with the teaching style. In addition, the guided inquiry technique is usually focused on shorter-term problems (i.e., solving a small subset of problems in a class period to focus on specific student learning outcomes). In contrast, the problem-based approach is usually focused on longer-term problems (i.e., the students work on a large problem for the entire term, and the instructor serves as a facilitator to help the students when needed). The project-based technique requires that the students complete a project, usually with a defined final deliverable (de Graaf and Kolmos 2003). In civil engineering, capstone senior design courses are often project-based. The casebased technique requires students to analyze a case history (Kardos and Smith 1979; Srinivasan et al. 2012). The discovery-based technique is similar to the guided inquiry technique, except the students are largely self-directed (Bruner 1961). Prince and Felder (2006) recommend against the discovery-based technique for undergraduate courses, following findings from Singer and Pease (1978) that the guided inquiry technique was more effective for helping students learn new concepts. Finally, the just-in-time teaching approach requires students to take assessment quizzes before class, and the instructor to adjust the instruction according to difficulties the students are having with the material (Novak et al. 1999).

We focused on the guided inquiry inductive learning approach to increase the impact of our work, because Prince and Felder (2006) stated that it is the easiest inductive learning approach. Accordingly, it is the most appropriate learning technique for inexperienced or traditional instructors to try first.

\subsection{MOTIVATION}

\subsection{INTERACTIVE CLASSROOM ENGAGEMENT}

Evidence suggests that engaging students in the learning process during a presentation - that is, motivating the students to be interactive learners - is an effective method for changing their conceptual understanding (e.g., Hake 2002; Prince 2004; Chi 2009). Interactive learning requires students to do more than passively listen. Interactive learning requires activities such as writing, discussion, and tactile problem-solving, and all the aforementioned activities engage students in higher-order thinking tasks such as analysis, synthesis, and evaluation.

Chi (2009) studied the effectiveness of three different active learning environments: active, constructive, and interactive. An active-learning environment engages students in simpler individual activities, such as taking notes or highlighting passages. A constructive-learning environment engages students in activities that are conceptually more difficult than the material students have recently learned; as an example, students may be required to combine multiple concepts to solve more complex problems without obvious solutions. Finally, in an interactive environment, students perform constructive activities in groups. This operationalization of active-learning environments is important, because Chi (2009) found that interactive 
activities have a greater effect on student learning outcomes than those of constructive activities, which in turn have a greater effect than those of simpler active learning activities. As defined previously, we do not include Chi's definition of an active-learning environment into our definition; rather, we include the levels designated as constructive and interactive. A critical component of the active-learning classroom is the difficulty of the activities in which students engage. If the activities are too simple, then students will not work together (Brown et al. 2009); if they are too difficult, then students will become frustrated and give up.

To maximize student-learning outcomes in transportation geotechnics, we will promote interactive engineering classrooms. Students will be tasked with working together on problems leveraging the transportation geotechnics DLM that are more difficult than those they have previously solved, and that require the synthesis of multiple concepts from transportation and geotechnical engineering.

\subsection{Desktop Learning Modules}

Traditional teaching methods are continuing to produce engineering students who have difficulty understanding conceptual concepts (Burgher et al. 2013). DLMs are being developed to create an educational experience in engineering that develops hands-on and problem-solving skills for students (Arasteh et al. 2013). One example of a DLM in development, described by Arasteh et al. (2013), focuses on the simulation of cell separation in bioengineering courses. This particular DLM contains scaled down processes like shell and tube or double pipe heat exchangers for chemical engineering and hydraulic flow channels for civil engineering.

The incorporation of DLMs that target misconceptions have been demonstrated to reduce conceptual difficulties experienced by engineering students (Burgher et al. 2013). In addition, Paul et al. (2009, pg. 3) found that "DLMs are well accepted by students and have been shown to enhance student learning." As a specific example, DLMs have been successful in reducing the frequency of misconceptions related to pressurized pipe flows through a straight pipe, shallow bend, and $90^{\circ}$ miter bend, and a straight pipe with an $180^{\circ}$ bend (Burgher et al. 2013). A control-group design was implemented with the DLM as the treatment in a civil engineering water resource class on open-channel flow, flow control and measurement. It was found that the concept inventory performance for the group that used the DLM improved 52.1\% over pre-test results (Paul et al. 2009).

Additionally, 98 percent of students surveyed stated that hands-on learning with the DLM helped them recall important facts with greater ease, and 93 percent stated that they had a better conceptual understanding of the topic (Paul et al. 2009). However, another study using the fluid mechanics DLM indicated that no statistical difference in gains between the control and treatment groups (Peterson et al. 2012). The authors concluded that there was a trend in improved abilities to describe abstract concepts on the material one week after the experiment (Peterson et al. 2012).

Another study evaluated the effectiveness of DLMs with associated activities and assessments (DLM\&A) implemented in undergraduate engineering classes (Brown et al. 2014). Both the control and experimental group responded to pre- and post- assessments. The control group participated in eleven interactive lecture sessions on open channel flow while the experimental group participated in nine lectures and two 50-minute sessions with the DLM\&As (Brown et al. 2014). "The experimental group registered a gain of 0.57 out of 1.0 possible, with 70 percent of the students achieving minimum competency, compared to a respective 0.26 gain and 39 percent competency for the control group (Brown 
et al. 2014)." The study concluded that there was strong evidence that student's conceptual understanding increased significantly when the DLM\&A were incorporated into their learning environment (Brown et al. 2014).

As previously described, DLMs, such as the transportation geotechnics DLM developed in this project, can improve the conceptual understanding of engineering students.

\subsection{Transportation Geotechnics}

Transportation geotechnics is a broad field, which is at the intersection of geotechnical engineering and transportation engineering. Fundamentally, transportation geotechnics is the study of how transportation infrastructure (e.g., roadways, bridges) and geotechnical materials (e.g., rock, soil) interact. Important subtopics within the field of transportation geotechnics include, but are not limited to: (1) understanding the geotechnical properties of soils, rock, or soil mixed with novel reinforcing material used a subgrade material for transportation infrastructure, (2) the use of geosynthetics on, under, or around transportation infrastructure, (3) building embankments for transportation infrastructure such as heavy rail, (4) the relationships between the geometric design and the surrounding subgrade materials, (5) pavement design, and (6) understanding how soil and transportation infrastructure interact during natural hazards. As an example, one particular concern during natural hazards is a phenomenon called soil liquefaction (Seed 1979). During soil liquefaction, the soil behaves as a liquid, and thus, transportation infrastructure built atop the liquefiable soil cannot be reliably supported. Figure 1a shows an example of the devastating effects of soil liquefaction on transportation infrastructure. Ultimately, the goal of transportation geotechnics is to design and construct safer, longer-lasting, and economically viable transportation infrastructure.

\subsection{METHODS}

\subsection{PROJECT SCOPE}

This project was primarily concerned with improving student understanding of how soil and transportation infrastructure interact during natural hazards. Based on this focus, our transportation geotechnics DLM focused on the concept of an earthquake response spectrum (Housner 1941, Biot 1941). The response spectrum is an engineering design tool that tracks the response of simplified structures to external loading. For instance, a displacement response spectrum may track the maximum displacement of many different bridge decks or elevated roadways during earthquake loading. The power of the response spectrum as a design tool is significant. It enables engineers to investigate how a wide variety of transportation infrastructure systems will respond during an earthquake motion. That information can help to specify preferable alternative designs.

Based on popular structural dynamics textbook organization (e.g., Clough and Penzien 1975; Chopra 2011; Humar 2012), as well as the authors' experience, response spectra concepts are commonly taught using a deductive approach. First, the governing equation of motion and dynamic properties for a single-degreeof-freedom oscillator, which is a simplified model of the more complex transportation infrastructure components, is given to the students. The equation of motion is often derived using an $\mathbf{F}=m \mathbf{a}$ argument (i.e., Newton's second law of motion). Second, the students learn how to solve the equation of motion, with the given dynamic properties, using a numerical methods technique, such as the central difference method. Finally, students are shown how to plot a response spectrum with the equation of motion solution they developed in the second step. 
Figure 2 proposes one possible application of inductive learning for teaching response spectra. Students first use a response spectrum device (RSD) to understand how earthquake loading affects transportation infrastructure. Students are shown physically how complex transportation infrastructure can be simplified to single-degree-of-freedom systems, and more importantly, students are shown physically the limitations of this modeling assumption. The students take measurements from the DLM, and then they plot those measurements to discuss trends and observations. From the measurements, students develop the underlying theory with each other facilitated by the faculty member (i.e., the guided inquiry approach), and then the approach follows the deductive approach outlined in Figure 2 (as steps $c, d$, and e) with one very important difference. In the final step, when the response spectrum is plotted, the measurements taken during the DLM demonstration can be plotted directly on the theoretical response spectrum. Accordingly, students can further discuss why the theory and the measurements do or do not match.

According to the foregoing discussion, the inductive approach, when considering the response spectrum example, has several key advantages. The primary advantage is that limitations of methods and deviance between theory and measurements can be discussed, which is a critical element to understanding engineering design.

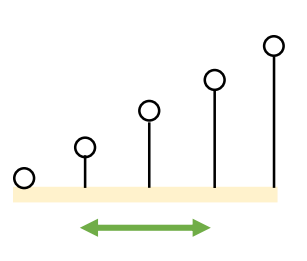

(a)

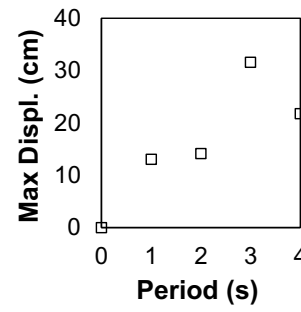

(b)

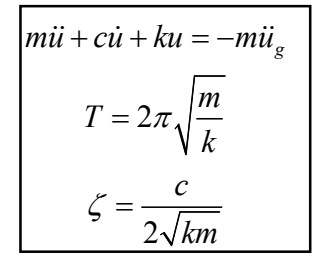

(c)

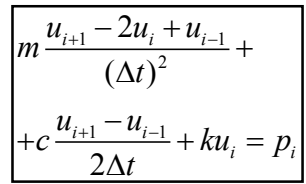

(d)

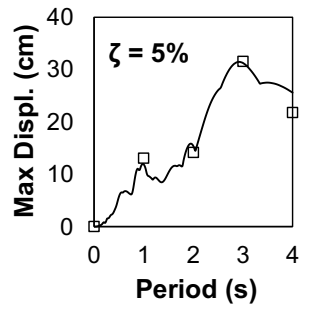

(e)

Figure 2. The inductive approach for teaching response spectra: (a) a DLM for simulating response spectra, (b) physical measurements from the DLM, (c) the theory behind the DLM and measurements; i.e., equation of motion and vibrational properties, (d) the numerical method used to solve the equation of motion, and (e) the displacement response spectrum.

We developed a DLM to promote the inductive learning of response spectra for transportation infrastructure. We chose to develop and implement the response spectrum device first because of the numerous possible alternatives and it was the most straightforward design, could be developed with the least expense, and would be widely applicable across a variety of civil engineering sub disciplines. Three graduate students, one in transportation engineering, one in geotechnical engineering, and one in geomatics engineering worked with the machine shop in the college of engineering to design high quality physical models and instrument the physical models. CE faculty members will implement these models in several civil engineering classes at Oregon State University staring in the Fall Quarter of 2016.

\subsection{PROTOTYPE DEVELOPMENT}

The first developed DLM visually demonstrates the response spectrum of a structure subject to earthquake loading, RSD. The RSD is a series of vertical springs attached to a rigid, moveable plate (Figure 3 right) with a spherical weight (Figure 3 left) attached to the uppermost location of each spring. The apparatus simulates a structure of a certain stiffness, modeled by the spring, and equivalent lumpedmass, modeled by the spherical weight, to predict the seismic response of a structure. 

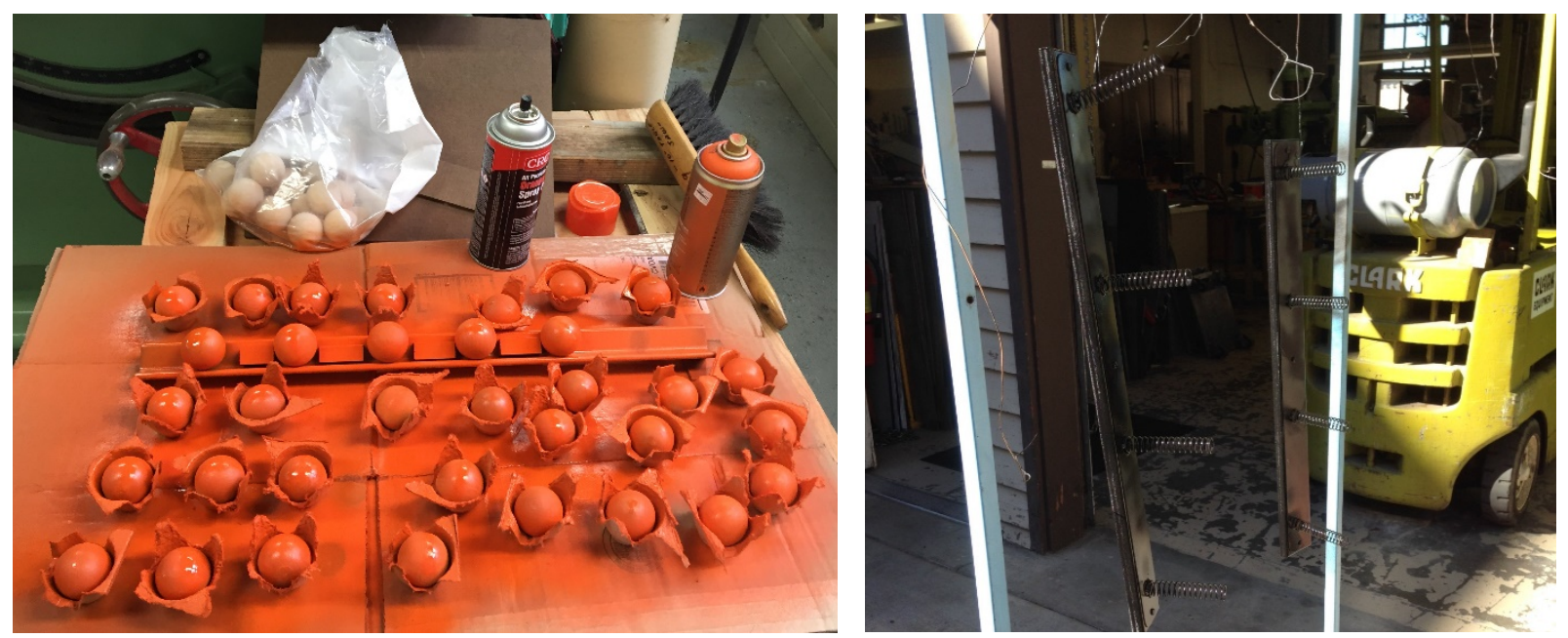

Figure 3. Development of finalized RSDs. Spherical weights were painted (left) and later applied to springs that had been welded to plates and painted (right).

To verify the proper selection of the springs used in the model, several iterations of RSD prototypes were developed with varying spring length and stiffness. The springs were required to be stiff enough to support the applied weights, but slender enough to act as a "weightless member" with respect to the modeled lumped-mass. Once the optimized spring arrangements were determined, various weights were applied to simulate lumped-mass point loads. A sample of the various spring and weight arrangements investigated are shown in Figure 4.

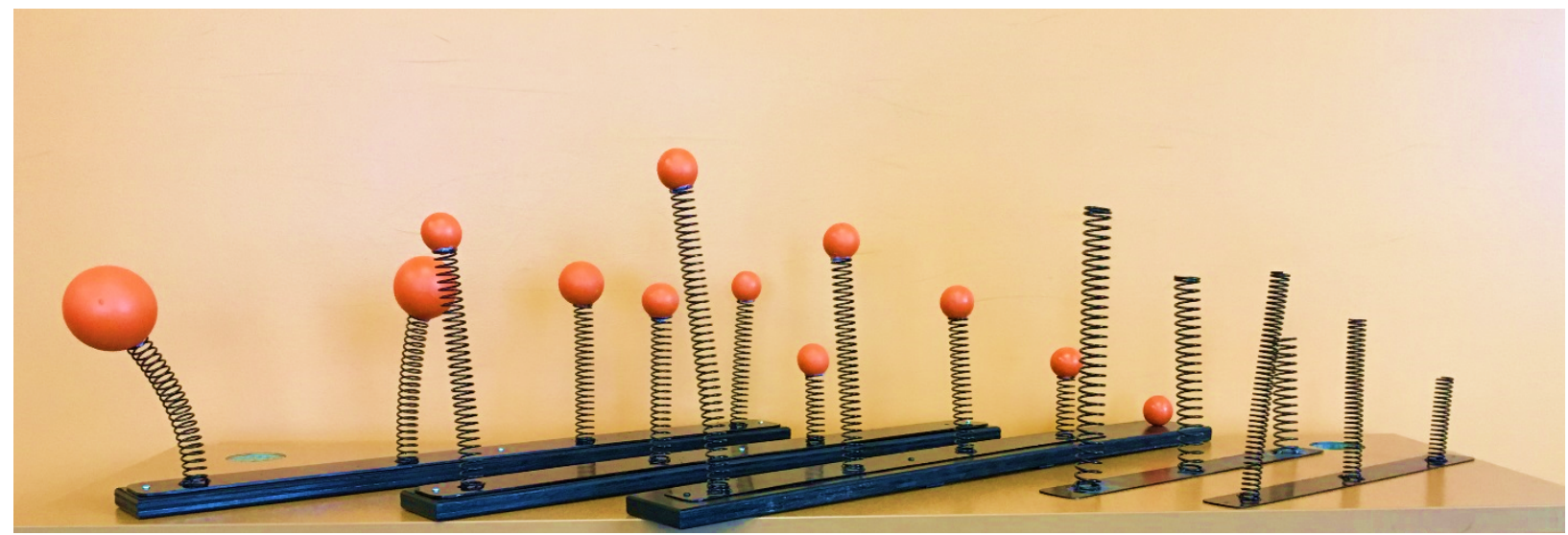

Figure 4. Various spring and weight arrangements investigated to determine the best configuration for the finalized RSDs.

Ultimately, two unique RSD models were produced: one that demonstrates the effect of varying stiffness on the response of a system, and the other demonstrates the effect of varying mass (Figure 5). The specifications of the two developed RSD components are detailed in Table 1. The information given in Table 1 could be useful for calculations necessary in classroom exercises. It should be noted that additional materials were used such as spray paint and a rigid base that were not included in the specifications. The completed RSDs without instrumentation cost around \$25 for necessary materials plus labor expenditures. 

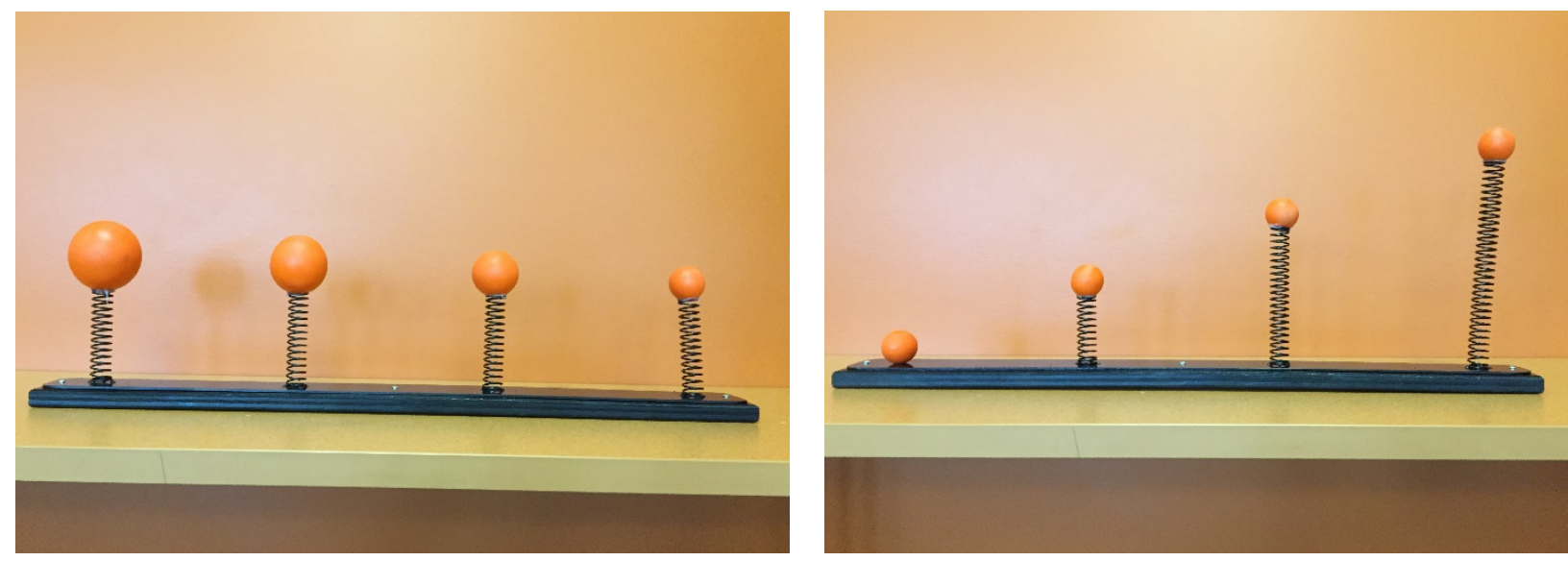

Figure 5. Finalized RSDs without instrumentation. The model on the left demonstrates the effect of varying structure weights and on the right the effect of varying stiffness of the structure.

The selection of the finalized materials to be implemented into the RSDs led to the creation of 14 finalized models. A production plan was executed between several graduate students and the machinist for the engineering department at OSU.

Table 1. Specifications of RSD components. The noted size and mass for each component is estimated from a sample set of RSD supplies. Each finalized model will have slightly different specifications due to construction processes.

\begin{tabular}{|c|c|c|c|}
\hline Object & Size & Mass (g) & Cost \\
\hline Small weight & 1.5" diameter sphere & 20.8 & $\$ 1.58$ ea. \\
\hline Medium weight & 2.0" diameter sphere & 47.7 & $\$ 3.45$ ea. \\
\hline Large weight & 2.5" diameter sphere & 84.4 & $\$ 8.40$ ea. \\
\hline Extra-large weight & 3.0" diameter sphere & 141.9 & $\$ 7.99$ ea. \\
\hline $\begin{array}{l}7 / 8^{\prime \prime} \text { outer diameter } \\
\text { spring }\end{array}$ & 1" length segment & $\begin{array}{l}\sim 5.5 \text { (average for } 1^{\prime \prime} \\
\text { segment*) }\end{array}$ & $\begin{array}{l}\$ 0.13 \text { per } 1 \text { " length } \\
\text { segment }\end{array}$ \\
\hline
\end{tabular}

*Should add 3 grams to estimated spring mass to account for finished ends (1.5 $\mathrm{g}$ for each end)

Following the development of the two RSDs without instrumentation, initial classroom trials were executed. A structural dynamics course (CE 534) and static and dynamic soil behavior course (CE 577) utilized the RSDs to demonstrate phenomena already outlined in the respective course curriculum. The natural period of a modeled structure was easily demonstrated by imposing an initial displacement on a selected spring and weight and observing the resonant frequency. The respective natural period could then be compared to the remaining spring and weights and inferences were made about the trends as a function of the changing variables. Another exercise to demonstrate earthquake loading applied a cyclic motion to the base of the apparatus and the spring and weight movements were observed. The imposed cyclic motion exposed the element that was most excitable. This observation led to the conclusion that the respective period of the applied cyclic motion corresponded to the natural period of the most excitable spring and weight component. As the frequency of the applied cyclic loading increased, additional modes could be observed in the more slender elements. The damping of the system was another observable phenomenon that was relevant to the structural response of earthquake motions.

The visible deformations and physical movements of the system are useful for aiding students in performing a qualitative analysis and develop an intuition for the response trends. The instrumentation 
of the RSD with three axis accelerometers at the center of each of the spring masses provides a data product which students can use to extend their qualitative intuition into more advanced equation and quantitative hypothesis testing. The accelerometer data can also be processed to estimate velocity and position to assess the complete response spectrum of the system. It should be noted that acceleration is measured directly through the instrument package, and while velocity and position can be calculated through integration, noise in the acceleration measurement propagates with this integration. A preliminary instrumented RSD along with the triaxle response data is shown in Figure 6.
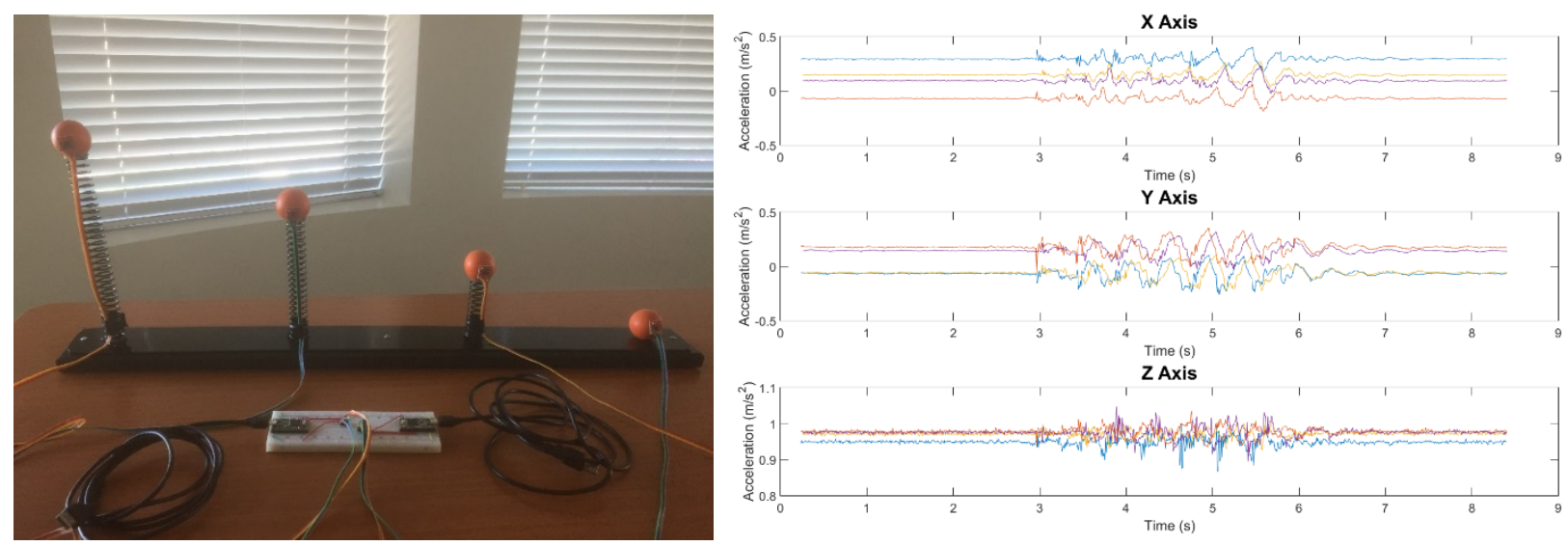

Figure 6. The preliminary instrumented RSD (left) is shown with a sample of 3-axis accelerations exerted on each of the spring masses plotted with time (right). Accelerometers fixed to the model weights record and plot the response when the device is subjected to motion.

\subsection{FINAL RSD}

The final RSD instrumentation and acquisition software programming language were selected to ensure an open-source and low cost system so that it could be adopted by future collaborators without a large financial investment. The hardware cost of a single electronics package was $\$ 128.48$, and all of the software was free and open source. A GUI and command line tool were both developed for data acquisition using the Python programming language.

\subsubsection{Electronics Package}

The electronics package is designed to log data from four, three axis accelerometers directly to a laptop of desktop PC. The accelerometers are initialized using $\mathrm{I}^{2} \mathrm{C}$ protocol and logged using two Teensy 3.2 microcontrollers, which are programmed using the Arduino IDE. The data is then transmitted from each of the microcontrollers via USB to the data logging computer via a Serial Port. The protoboard diagram for the electronics package are shown in Figure 7. 
PROTOBOARD: BOTTOM

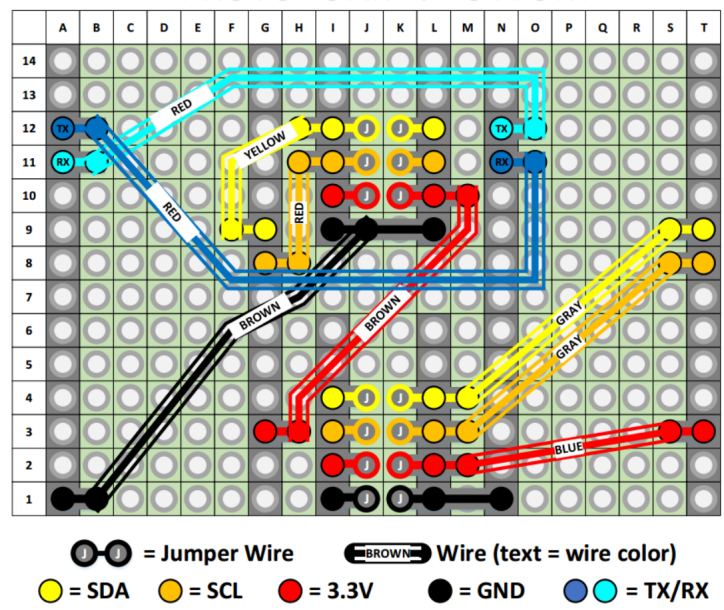

PROTOBOARD: TOP

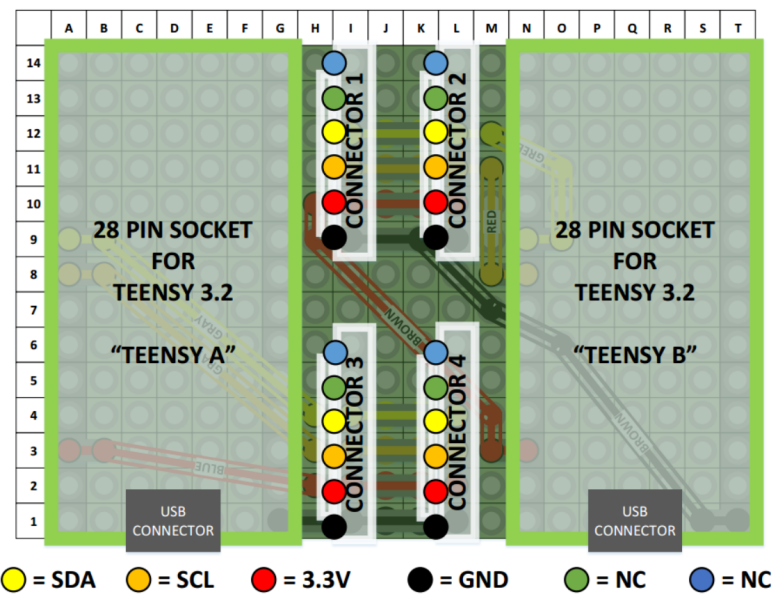

Figure 7. The protoboard design for each electronics package is used to interface the microcontrollers with the accelerometer connectors.

The microcontrollers and accelerometers are connected to the protoboard using sockets and connectors respectively in order to maintain modularity of the system and improve the ease of debugging. This makes the system easier to transport between the classroom and the lab by enabling the disassembly of the system so that the electronics may be carried separately to reduce the risk of damage. The itemized components list for each electronics package is shown in Table 2.

Table 2. The components for the electronics package were selected to minimize the cost of the overall system.

\begin{tabular}{|l|l|l|c|}
\hline Part & Unit Cost (\$) & Quantity & Total Cost (\$) \\
\hline Accelerometer & 9.95 & 4 & 39.80 \\
\hline Teensy 3.2 microcontroller & 19.95 & 2 & 39.90 \\
\hline micro-USB cable & 4.95 & 2 & 9.90 \\
\hline Ribbon Cable & 4.95 & 1 & 4.95 \\
\hline USB Hub & 6.99 & 1 & 6.99 \\
\hline 10 Prototype Boards & 3.95 & 1 & 3.95 \\
\hline 28 Pin Socket & 1.25 & 1 & 1.25 \\
\hline 6 Wire Assembly & 1.95 & 4 & 7.80 \\
\hline 1/16" Heat Shrink (10ft) & 6.99 & 1 & 6.99 \\
\hline Jumper Wires & 6.95 & 1 & 6.95 \\
\hline Total & & & 128.48 \\
\hline
\end{tabular}

\subsubsection{Software}

The serial data is transmitted to the laptop or desktop PC via two USB COM ports. This data may be logged using numerous software packages which will log COM port data streams, however all of the raw data would be logged to two separate text files. In order to make a more seamless data logging experience for 
the user, two binary executables were packaged from Python code to log the data to a single text file. Python was selected as it is free, cross platform, open source programming language commonly used by engineers. It also has a large online community, numerous open source packages, and thorough documentation.

The first Python script is a command line tool which detects a connection to the COM ports and multiplexes the data from both serial ports into one data file. The data in this file can then be postprocessed to visualize accelerations and calculate numerous response parameters. The command line tool is meant as a very barebones tool for users who only care about logging data, and not visualizing the data in real time.

A more elaborate Python script was designed to generate a Graphical User Interface (GUI), which is used to visualize the data in real time while logging the data. A screenshot of the tool is shown in Figure 8. The foundation for the GUI is built using the cross-platform modules PyQt and pyqtgraph, so that linux and mac users will also be able to run the software. The GUI contains an "interactive legend" which enables users to customize which variables are being plotted using the checkboxes. The user can also vary the color and line width of the variables being plotted to optimize a custom visualization in a classroom setting to assist in highlighting a specific response. Control of data acquisition and saving is performed by clicking the "Start", "Stop", and "Save" buttons in the lower right of the GUI.
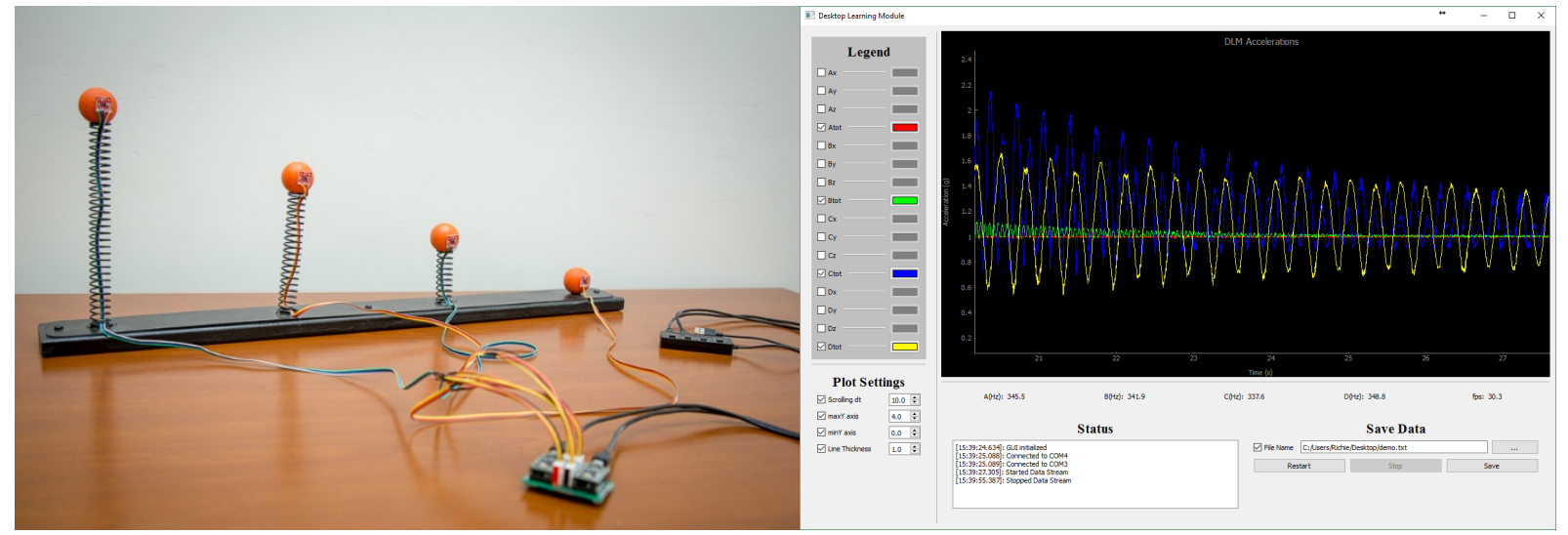

Figure 8. Finalized DLMs with instrumentation (left), and the Python GUI for data acquisition visualizes the accelerometer data in real time (right).

Both the command line tool and the GUI have been packaged to a windows binary executable so that users can run the software with no external dependencies or installation required. Further documentation on functionality and documentation of the acquisition software are available on the Github repository described in Section 4.2.

\subsection{RESULTS OF IMPLEMENTATION}

To test the efficacy of the DLMs, we collaborated with three instructors (A, B, and C) at Oregon State University, who agreed to use the DLMs during the 2016-17 academic year. The instructors used the DLMs in three courses, herein labeled I, II, and III. Courses I and III are required courses, and Course II is an elective course. Course I is an upper-level undergraduate course that had an enrollment of fifty students, and Courses II and III are graduate courses that had enrollments of fifteen graduate students and nine 
students (seven graduate students, two undergraduate students), respectively. The three instructors developed lesson plans to use the DLMs independently and without our input. A graduate student researcher trained the instructors to use the DLMs, observed the in-class use of the DLMs, and then conducted post-class interviews with the three instructors (Buker 2017).

Through classroom observation, we found that the three instructors implemented different in-class learning activities to promote inductive learning approaches with the DLMs. In particular, all three instructors used the DLMs to create response patterns that the students could observe, and the observations helped the students develop formal theory with guided inquiry from the instructors. Moreover, instructors $A$ and $B$ allowed students to operate the DLM and had the students hypothesize the response of the DLMs to different input motions before using the DLMs. Finally, instructors B and C utilized the Python scripts to further the students' understanding of the underlying theory by showing how response trends change with changes in frequency and amplitude of excitation in real time. Notably, instructors $\mathrm{B}$ and $\mathrm{C}$ defaulted to a deductive learning approach by providing the students with background information on response spectra before using the DLMs.

We also found that the three instructors implemented in-class learning activities to promote interactive classrooms. In particular, all three instructors required the students to participate in a think/pair/share activity before using the DLMs to predict the response patterns, and all three instructors asked students questions before using the DLMs and encouraged the students to ask questions before, during, and after each DLM demonstration. In addition, as mentioned above, instructors A and B allowed the students to operate the DLM.

We conducted post-implementation interviews with all three instructors to gather more information. Importantly, all three instructors found that students were more actively engaged in the classroom when they used the DLMs (as compared to previous lessons when the instructors covered similar content using a deductive learning approach). In addition, all three instructors agreed that they would use the DLMs again in the future. Finally, using the DLMs sparked the instructors' imaginations for creating and using other DLMs in future course offerings.

\subsection{CONCLUSIONS}

\subsection{PHYSICAL MODELS}

The research team constructed seven pairs of RSD models (one model of constant mass, varying stiffness and one model of varying mass, constant stiffness). Five Civil Engineering professors (Dr. Tom Miller, Dr. Judy Liu, Dr. Michael Scott, Mr. Kenny Martin, and Dr. Andre Barbosa) were each provided with a pair of RSD models. These models are currently planned for implementation in a variety of relevant civil engineering classes including: structural dynamics (CE 534 W17 Scott), structural mechanics (CE 531 F16 Yim), structural stability (CE 533 Sp17 Miller), stat/dyn soil behav. (CE 577 F16 Mason), geotech. eq. engr. (CE 578 F16 Mason), masonry design (CE 582 W17 staff), seismic design (CE 589 F16 Miller),

\subsection{SOFTWARE}

The development and instrumentation of the RSDs has been thoroughly documented on Github (https://github.com/OSU-Geomatics/OregonState DLM) and shared with academic colleagues to serve as the central repository for access to and continued development of the RSDs. Github serves as a platform for the collaborative, open-source development of tools that will specifically be used to improve the 
usefulness of the RSDs via visualization and data analysis algorithms. This collaboration will augment the usefulness of the system in a classroom environment, and provide a platform to ensure the longevity and functionality of the RSD models.

\subsection{SCHOLARLY PRESENTATIONS}

Members of the research team have made several scholarly presentations communicating the development of the RSDs to different audiences. These presentations include:

\section{- Geocongress award winning video}

Prior to instrumentation, a varying stiffness RSD was utilized in the $2016 \mathrm{Geo} /$ Structures Student Video Competition. The video created by civil engineering graduate students at OSU demonstrates earthquake loading on the RSD imposed with a shake table. The theory of the natural period of structures is introduced as a function of mass and stiffness followed by a demonstration which exposes the natural period of each spring and mass component. The setup is then modified to include the effects of a softer soil beneath the modeled structures by inserting Jello between the RSD and the shake table. The findings are then applied to a case study in which medium height buildings were affected greater than short or very tall buildings due to the effect of the natural period and earthquake excitation imposed on the structures. The video received first place in the competition.

https://www.youtube.com/watch? $\mathrm{v}=\mathrm{mGVqohQUa5s \& list=PLXHjEGSzGOPNRzOaGBdz8C}$ qOKgcBTYa-U\&index $=1$

\section{- $85^{\text {th }}$ Annual Pacific Northwest ASEE Conference Presentation}

Dr. David Hurwitz presented at the $85^{\text {th }}$ Annual Pacific Northwest ASEE Conference, "Focusing on Student Success" at Boise State University from March 31 to April 2, 2016. The presentation titled, "Development and Implementation of Physical Models for Transportation Geotechnics" took place on Friday, April 1, 2016 in Session B1, "Teaching Professional Skills 1". The audience for the session was primarily composed of faculty teaching a variety of engineering classes at universities, colleges, and community colleges in the Pacific Northwest. The Conference agenda can be found here: http://pnw.asee.org/Newsletters/2015/2016ASEEPNWConferenceSchedule.pdf.

\section{- $\quad$ OSU College of Engineering Graduate Research Expo}

The second annual Engineering Graduate Research Expo was hosted at the Portland Art Museum on Tuesday, March 1, 2016. Kamilah Buker and Rachel Adams were accepted to present the poster titled, "Development and Implementation of Physical Models for Transportation Geotechnics". Over 200 Oregon State University graduate students showcased their research at this venue that was opened to the general public. The details of the Graduate Research Expo can be found here: http://engineering.oregonstate.edu/gradexpo2016

\subsection{FUTURE WORK}

The successful response spectrum devices produced will be modified, such as the addition of an instructional shake table and additional instrumentation, to refine the demonstrations and generate useable data for student analysis. In addition to the current collection of DLMs, future models could be developed to demonstrate phenomena such as sedimentation, volumetric changes in soil, and soil to structural and transportation system interactions during liquefaction. 


\subsection{REFERENCES}

1. Arasteh, A., Clark, C. M., Wie, B. J. Van, Abu-lai, N. I., Adesope, O., and Brown, S. A. (2013). "Work in Progress:Development of Hands-on Desktop Learning Modules for Bioengineering Courses." $120^{\text {th }}$ ASEE Annual Conference \& Exposition.

2. Biot, M. A. (1941). "A Mechanical Analyzer for the Prediction of Earthquake Stresses." Bulletin of the Seismological Society of America 31, 151-171.

3. Bishop, J. L. and Verleger, M. A. (2013). The Flipped Classroom: A Survey of the Research. $120^{\text {th }}$ ASEE Annual Conference \& Eposition Conference Compendium.

4. Brown, S., Easley, A., Monfort, D., Adam, J., Van Wie, B., Olusola, A., Poor, C., Tobin, C., and Flatt, A. (2014) "Effectiveness of an Interactive Learning Environment Utilizing a Physical Model." ASCE Journal of Professional Issues in Engineering Education.

5. Burgher, J. K., Finke, D., Wie, B. J. Van, Adesope, O., Brown, S. A., and Atkinson, J. W. (2013). "New Hands-on Fluid Mechanics Cartridges and Pedagogical Assessment." $120^{\text {th }}$ ASEE Annual Conference \& Eposition ASEE.

6. Chi, M. T. H. (2009). "Active-Constructive-Interactive: A Conceptual Framework for Differentiating Learning Activities." Topics in Cognitive Science, Volume 1, 2009, pp. 73-105.

7. De Jong, T., Linn, M. C., and Zacharia, Z. C. (2013). "Physical and Virtual Laboratories in Science and Engineering Education." Science 340, 305-308.

8. Felder, R.M and Silverman, L.K. (1988) "Learning and Teaching Styles in Engineering Education," Engr. Education, 78(7), 674-681.

9. Housner, G. W. (1941). "Calculating Response of an Oscillator to Arbitary Ground Motion." Bulletin of the Seismological Society of America 31, 143-149.

10. Hurwitz, D., Brown, S., Islam, M., \& Daratha, K. (2013). Mental Models of Students and Practitioners in the Development of an Authentic Assessment Instrument for Traffic Signal Engineering. $120^{\text {th }}$ ASEE Annual Conference \& Exposition Conference Compendium.

11. Hurwitz, D., Brown, S., Islam, M., Daratha, K., \& Kyte, M. (2014). Traffic Signal System Misconceptions across Three Cohorts: Novice Students, Expert Students, and Practicing Engineers. Transportation Research Record: Journal of the Transportation Research Board Issue 2414, 52-62.

12. Islam, M., Hurwitz, D., Brown, S. (2014). Identifying Traffic Signal System Misconceptions of Students and Practicing Engineers to Develop a Traffic Signal Concept Inventory. $121^{\text {th }}$ ASEE Annual Conference \& Exposition Conference Compendium.

13. Kyte, M., Dixon, M., Abdel-Rahim, A., and Brown, S. "Process for Improving Design of Transportation Curriculum Materials with Examples." Transportation Research Record, No. 2199, 2010, pp. 18-27

14. Paul, C. D., O'Neil, M. B., Penney, W. R., Wie, B. J. Van, Golter, P. B., Beitle, R. R., Clausen, and E. C., and Martin, R. E. (2009). "Desktop Learning Module Heat Exchanger Performance." 2009 Midwest Section Conference of the ASEE.

15. Peterson, K. L., Compere, M. D., Allam, Y. S., and Wie, B. J. Van. (2012). "A Fluid Flow Characerization Device for an Educational Desktop Learning Module." Proceedings of the ASME IMECE.

16. Seed, H. B. (1979). "Soil Liquefaction and Cyclic Mobility Evaluation for Level Ground During Earthquakes," Journal of Geotechnical Engineering 105(GT2), 201-255.

17. Silverthorn, D. U. (2006). "Teaching and Learning in the Interactive Classroom." Advances in Physiology Education, 30, 135-140. 
18. Visco, D.P. (2011) "Student Designed Desktop Modules in a Thermodynamics Course" American Society for Engineering Education (ASEE) Annual Meeting Compendium.

19. Wie, B. J. Van, Thiessen, D. B., Marc Compere, E.-R., Toro, X., Adam, J. C., Brown, S. A., Easley, A. P., Li, X., Lee, K., Colpan, M., Gray, K. T., Garret, B., Reynolds, S. R., Golter, P.B., and Adesope, O. (2012). AC 2012-5271: MULTI-DISCIPLINARY HANDS-ON DESKTOP LEARNING MODULES AND MODERN PEDAGOGIES." ASEE. 